\title{
L'innovation lexicale dans le discours journalistique Le cas des chroniques Tranche de Vie et Raïna Raïkoum
}

\section{Résumé :}

La question de l'innovation lexicale est évidemment centrale en Sciences du Langage. Étant donné que toute langue suit la dynamique sociale, le lexique des langues pratiquées en Algérie est en permanente évolution.

Dans la presse écrite, on observe des créations lexicales qui touchent à de nombreux domaines de la vie quotidienne. Notre recherche tente d'éclairer ce phénomène, de montrer que les néologismes journalistiques ne sont pas dus au seul besoin de désigner de nouvelles réalités mais que leur éclosion dans ce type de discours est reliée à différents facteurs et doit être étudiée en prenant en considération les conditions dans lesquelles ils étaient produits. En conclusion, nous soulignons que ces néologismes sont un outil linguistique essentiel dans le discours journalistique algérien.

Mots-clés : néologismes- analyse de discourspresse écrite - conditions d'énonciation.

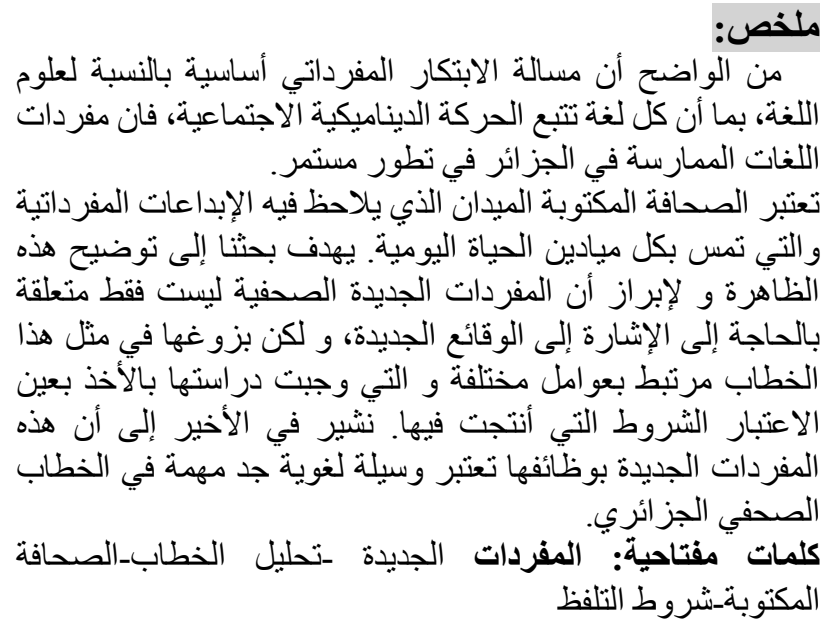

\author{
Sana ADASI \\ Département des lettres et langue française \\ Université des frères Mentouri \\ Constantine 1
}

\section{Introduction : \\ Les \\ productions} néologiques effectuées par des journalistes, dans un contexte donné, constituent un acte d'énonciation qui se définit selon BENVENISTE $^{(1)}$ comme « une mise en fonctionnement de la langue par un acte individuel d'utilisation». Selon les principales théories de la pragmatique linguistique, tout énoncé est porteur de sens, pour pouvoir le déterminer, le chercheur doit s'intéresser à ses conditions d'usage et à son contexte d'énonciation. 
Relèveront donc de l'énonciation des éléments tels que l'énonciateur, son statut, son rôle, ainsi que la relation qui s'instaure entre lui et le destinataire de son message à travers l'énoncé.

Tout texte est le résultat d'un acte énonciatif produit dans une situation précise, la prise en compte des conditions de sa production est nécessaire afin de pouvoir accéder à son sens, en particulier pour vérifier si le recours à la création lexicale dans la presse écrite vise à faire agir le lecteur, à susciter chez lui des comportements ou tout simplement à faire aboutir une intention de communication. Il devient donc indispensable de recentrer notre intérêt, dans la présente recherche, sur les conditions d'énonciation des néologismes.

Afin de mieux saisir les spécificités des textes dans lesquels apparaissent les néologismes, nous exposerons quelques informations relatives au journaliste et à son statut, aux destinataires (les lecteurs) et à leurs attentes. Nous nous intéresserons, également, à la visée de communication ainsi qu'aux caractéristiques des messages véhiculés par le support de transmission, qui est la presse écrite, en mettant l'accent sur les spécificités de l'écriture journalistique.

Notre analyse a été menée sur un ensemble de néologismes relevés dans deux chroniques d'un journal algérien francophone qui est le Quotidien d'Oran ${ }^{(2)}$, et ce pendant la période s'échelonnant du 01 / 07 / 2007 au 30 / 09 /2007. Le nombre des néologismes collectés ${ }^{(3)}$ est de 140 lexies. En voici quelques exemples :

- Isklamiates (02/ 07/ 2007), Aïn-coup-de-pieds (05/ 07/ 2007).

- Ânarchie (05/07/2009), Union des Msarines Arabes ${ }^{(4)}$ (08/ 07/ 2007).

- Fast-think (15/ 07/ 2007), paysement (21/ 07/ 2007).

- Aïn Part (23/ 07/ 2007), sidi good year (28/07/ 2007).

- Aïn-Machin-Chouette (30/ 07/ 2007), nimportequoitisme (05 / 08/ 2007).

- Sauciologues (05 / 08/ 2007), festivals-rien (05 / 08/ 2007).

- Chouffer (08/ 08/2007), savoirerie (08/08/2007).

- Méfaits divers (15/ 08/2007), le fsède (25/ 08/ 2007).

- F quelque-chose (28/08/2007), indigénerie (03/ 09/2007).

- Bidon-douar (05/ 09/2007), popudégueulasse (09/09/2007).

- Mendiant-bizness (13/ 09/2007), resiester (16/ 09/2007), zabitants (25/ 09/2007).

Dans la chronique Tranche de Vie, animée par El Guellil (J.1) ${ }^{(5)}$, ont été recensées : 87 lexies néologiques $(62,14 \%)$. 
- Dans la chronique Raïna Raïkoum, animée par six (06) journalistes (J.2, J.3, J.4, J.5, J.6, J.7) pendant la même période de trois mois, nous avons collecté 53 lexies néologiques $(37,86 \%)$. Ces résultats sont représentés par le graphique suivant :

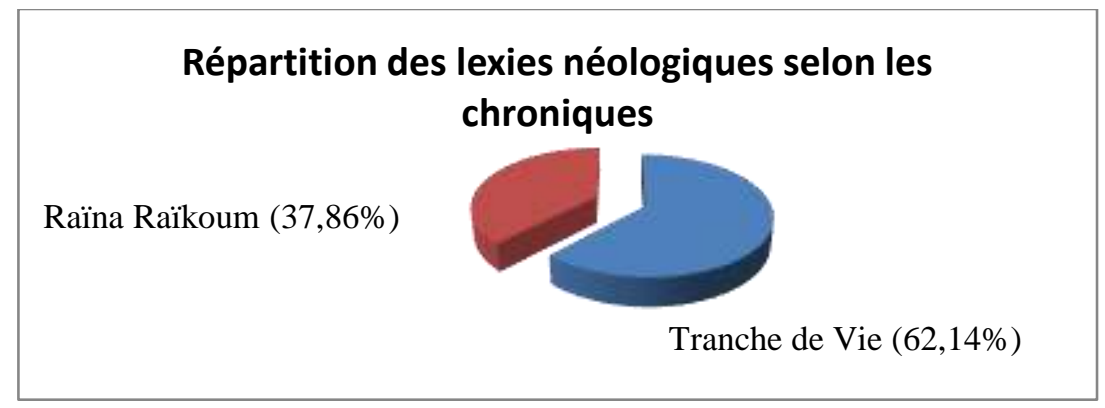

Le nombre des néologismes extraits de la chronique Tranche de Vie dépasse celui des néologismes repérés dans Raïna Raïkoum. Ce constat nous amène à nous poser la question suivante :

- Pourquoi les lexies néologiques relevées dans la chronique Tranche de Vie, imputables à un seul journaliste (qui est J.1), sont-elles plus nombreuses que celles extraites de la chronique Raïna Raïkoum, émises par six (6) journalistes ?

D'après ces résultats, on peut formuler comme hypothèse que les conditions d'énonciation dans lesquelles ont été émises les lexies néologiques relevées dans la chronique Tranche de Vie, favorisent l'émergence des créations lexicales, et c'est parce que ces conditions ne sont pas les mêmes dans les deux chroniques que nous avions pu remarquer une différence quantitative dans la production néologique. Les cas d'inégalité dans le nombre des néologismes émis par chaque chroniqueur nous ont conduite à examiner les causes de ces divergences et les différents facteurs pouvant influencer la néologie.

\section{Les conditions d'énonciation des néologismes :}

L'innovation lexicale s'effectue sans cesse sous l'effet de nombreux facteurs. Afin d'expliquer pourquoi ce phénomène linguistique prend une proportion importante dans le domaine de la presse, nous focalisons notre attention sur les conditions d'énonciation, qui se définissent en fonction du genre textuel -la chronique en tant que genre journalistique-, du locuteur intégré dans une situation institutionnelle donnée, du code linguistique, de l'objectif communicatif, etc.

\subsection{Conditions d'énonciation relatives à l'écriture journalistique :}




\subsubsection{La pluralité des codes linguistiques :}

Tout journaliste est censé avoir acquis un certain nombre de compétences lui permettant d'être usager autonome de l'outil linguistique. Maîtrisant deux ou plusieurs langues, il est producteur d'un discours plurilingue. Ce choix opéré par certains journalistes du Quotidien d'Oran -notamment le chroniqueur de Tranche de vie- et qui consiste à alterner, dans la quasi-totalité des cas, deux langues, semble conditionné par leur compétence. En prenant en considération le fait que les chroniqueurs, de ce même journal, sont des Algériens, le passage du français, la langue dans laquelle sont rédigées les deux chroniques Tranche de Vie et Rä̈na Rïkoum, à l'arabe algérien, langue maternelle, n'est pas aléatoire. Le fait que le journaliste, choisit, à un moment donné, de s'exprimer dans l'une ou l'autre des langues est très significatif. Selon BOYER $^{(6)}$, l'alternance de deux langues n'est pas forcément due à l'incompétence du locuteur bilingue, il note: «A travers ses choix de langues, à travers les marques transcodiques qu'ilutilise, c'est bien son identité - langagière, sociale-que l'individu exprime et (re)construit lors de chaque événement de communication ».

Le même auteur explique que l'un des résultats des contacts de langues est l'apparition de marques d'hybridation, ce sont les nouvelles formes linguistiques «qui n'appartiennent ni à la langue $A$, ni à la langue $B$ et qui peuvent avoir une durée de vie réduite au temps [d'une] seule conversation ou devenir habituelle pour [les] interlocuteurs. Ces formes constituent des créations réellement interlinguistiques $\gg^{(7)}$. La maîtrise de plus d'un code linguistique est un facteur important dans la création lexicale, le fait que les chroniqueurs ont pour langue maternelle l'arabe algérien, favorise directement la présence de termes arabes (emprunts) dans ces textes français, ainsi que le passage du lexique d'une langue à une autre rend de plus en plus probable l'émergence de lexies hybrides dans le discours.

\subsection{Conditions d'énonciation relatives au genre de la}

\section{chronique :}

\subsubsection{La chronique en tant qu'article de commentaire :}

Les textes à partir desquels nous avons fait l'extraction des néologismes, appartiennent à un genre particulier qui est la chronique. Il s'agit tout d'abord d'un genre journalistique se caractérisant par un mode d'écriture qui lui est propre. BROUCKER ${ }^{(8)}$ la définit comme étant: «L'article dans lequel une "signature » rapporte ses observations, impressions et pas réflexions au fil du temps passé. [...] C'est en quelque sorte un journal d'auteur à 
l'intérieur d'un journal de journalistes. L'auteur en question, qui d'ailleurs peut être ou ne pas être un journaliste, a ses propres critères de sélection et d'appréciation du ou des sujets dont il désire s'entretenir selon son humeur ».

Selon VOIROL ${ }^{(9)}$, la chronique appartient à la catégorie des articles de commentaire. Dans ce type de production journalistique, l'écrit est d'une grande subjectivité, le chroniqueur est amené à faire passer aux lecteurs ce qu'il voit et ce qu'il ressent lui-même. Le caractère subjectif de la chronique journalistique est fortement lié au fait que celle-ci appartient au genre de l'opinion, le chroniqueur a une opinion personnelle et la transmet au lecteur. Sa façon de lire l'actualité, de la commenter, fait surgir son positionnement subjectif par rapport aux différents événements. Ce sont alors les moyens linguistiques dont il se sert qui vont définir le caractère subjectif ou objectif de sa production langagière.

\subsubsection{Forme et taille de la chronique :}

Les deux chroniques journalistiques Tranche de Vie et Raïna Raïkoum sont deux articles encadrés et composés en caractère italique. Ils apparaissent dans chaque numéro du journal Le Quotidien d'Oran. Ayant conscience de l'espace réduit consacré à sa chronique, le chroniqueur rédige son article en se servant de tous les procédés stylistiques et linguistiques pour marquer son discours et maintenir l'attention des lecteurs. D'ailleurs un grand nombre de néologismes figurent dans les titres des chroniques. Ils ne peuvent être employés que pour inciter le lecteur à lire cet article de taille réduite.

Après avoir constaté les disparités numériques dans les résultats de la comparaison des néologismes émis dans les deux chroniques, nous avons décidé de comparer les conditions d'énonciation de la chronique Raïna Raïkoum avec celles de la chronique Tranche de Vie. La seule différence entre les deux chroniques réside dans deux facteurs : les registres de langue et l'acte humoristique.

\subsection{Registres de langue :}

MORTUREUX ${ }^{(10)}$ soutient l'idée que la qualité de la prononciation, de la syntaxe ainsi que le choix du vocabulaire sont les trois composantes de la langue qui permettent de définir les registres. A travers l'analyse des conditions d'émission des néologismes dans les chroniques, nous allons essayer de voir s'il y a une relation entre les différents registres de langue et la néologie. Nous allons nous intéresser aux trois composantes permettant l'identification des registres de langue dans notre corpus. 


\subsubsection{La qualité de la prononciation :}

DUCROT considère que "l'intonation n'a pas de réel équivalent à l'écrit car la ponctuation ne donne en vérité qu'une vague indication, qui permet de déterminer uniquement la fonction distinctive et démarcative ${ }^{(11)}$. A partir des textes écrits, il est donc impossible de se faire une idée exacte de la manière dont ceux-ci ont été prononcés. Etant donné que notre corpus est constitué de l'ensemble des chroniques journalistiques, ce critère s'avère impertinent pour la détermination des registres de langue dans ces textes.

\subsubsection{La syntaxe :}

Nombreux sont les néologismes qui, du point de vue syntaxique, témoignent de cette situation du non-respect $d u$ code. Ce sont les cas de conversion et de conversion verticale : le tout va bien (21/ 08/2007), le nimportequoitisme (05/ 08/2007), larbiner (23/07/2007), siester (16/09/2007), resiester (16/09/2009), le rani mdigouti (06/08/2007). Il est à noter que toutes ces lexies ont été relevées « uniquement» dans la chronique Tranche De Vie.

\subsubsection{Le vocabulaire :}

De manière générale, on constate que les journalistes dans les deux chroniques se servent de la langue écrite usuelle. Cependant, nous avons remarqué que certains emplois de la langue présentent des écarts par rapport aux formes standards du français. Comme il est tout à fait impensable de prétendre à l'exhaustivité sur ce point, observons quelques cas qui nous semblent significatifs :

\section{Tranche de Vie:}

- Nous avons relevé quelques mots qualifiés de familiers dans les dictionnaires : fric (02/08/2007), bagnole (09/09/2007), gosse (06/08/2007), gargote (26/08/2007), baggara, begri (09/09/2007), etc.

- Des expressions populaires : notamment en arabe dialectal. Nous en citons quelques exemples :

- «Devenir Charika gadra est leur but » (16/ 08/ 2007).

- « Tachrak el-foum $»(09 / 09 / 2007)$.

- «Abrassmmma, Oualou» (16/09/2007).

- «Zitna fi bitna» (08/ 08/2007).

- Les mots abrégés (ou troncations) sont employés généralement dans les situations de communication familière, le corpus des chroniques Tranche De Vie en compte 3 exemples : expo (25/08/2007), ado (09/08/2007), récup (23/09/ 2007).

Raina Raikoum : 
- Nous avions pu repérer le mot choper, qualifié de familier dans les dictionnaires.

- Dans cette chronique les journalistes se servent d'un français correct, les règles syntaxiques sont respectées et les phrases sont bien construites.

- Un seul exemple de troncation a été relevé dans cette chronique : protesta.

La création lexicale est différente d'un locuteur à un autre, on ne crée pas de la même manière selon la situation dans laquelle on se trouve. Les raisons pour lesquelles un locuteur recourt, à un moment donné, à la néologie, sont sans doute diverses, SABLAYROLLES ${ }^{(12)}$ admet que pour analyser ces causes, il faut s'intéresser à la fois à la position du locuteur dans l'interlocution ainsi qu'à son degré de maîtrise de la langue et enfin, au respect du code.

Ce que l'on peut tirer comme conclusion de cette comparaison, est que les marques associées au registre familier sont plus présentes dans la chronique Tranche de Vie que celle Raïna Raïkoum. Beaucoup de néologismes naissent dans les situations où les locuteurs pratiquent le registre familier. Ils apparaissent $\mathrm{du}$ fait du non-respect des règles du code linguistique.

\subsection{L'acte humoristique dans la chronique Tranche de Vie :}

Selon les conditions d'émission, les fonctions que l'on peut attribuer aux différents néologismes sont multiples. SABLAYROLLES ${ }^{(13)}$, qui emprunte à B.N. et R. GRUNIG les outils d'analyse proposés dans leur ouvrage La Fuite Du Sens, paru en 1985, soutient l'idée que c'est le faisceau causal du dire du locuteur qui est à l'origine de l'émergence des néologismes dans ses énoncés. Ces causes sont constituées d'un certain nombre de pressions selon lesquelles les néologismes se répartissent en plusieurs catégories : néologismes dévaluants, néologismes séducteurs, néologismes argument de vente, néologismes d'appel, néologismes ludiques, etc.

La visée de l'acte de communication pourrait influencer l'apparition des néologismes dans le discours, cependant, il est difficile d'établir une relation de causalité. L'humour, présent à travers certains néologismes résultants des jeux de mots dans la chronique, sert à faciliter la communication en créant des rapports de complicité et de familiarité entre l'émetteur et les destinataires. Par cette pratique, le chroniqueur -émetteur des néologismes -manie la langue pour faire plaisir aux lecteurs. Nous pensons que l'acte humoristique dans la chronique Tranche de Vie a favorisé, d'une manière ou d'une autre, l'émergence de certaines innovations lexicales. Il s'agit plus précisément des néologismes à fonction ludique ${ }^{(14)}$. Ceux-ci sont nécessaires à la production humoristique. 
D'après ce que nous avons remarqué, l'humour, dans la chronique Tranche de Vie, est fortement ancré dans la situation de communication. Les jeux de mots constituent un moyen privilégié pour obtenir cet effet, cela justifie le foisonnement des néologismes à fonction ludique dans cette chronique. EURARD $^{(15)}$ explique que: "l'écriture humoristique tend à manipuler le langage comme le lieu d'une activité ludique et poétique au sens de création, recréation et récréation ». L'humour entretient, avec la pratique linguistique, plusieurs types de relations. Nous avons extrait du corpus des unités linguistiques dont l'emploi vise à produire un effet humoristique :

Trop-laid (29/09/2007), terre-minus (29/09/2007), union des msarines arabes (08/ 08/2007), rencontre mille-feuille (08/07/ 2007), frites VIP (09/07/ 2007), l'an-seignement (28/07/2007), sauciologues (05/08/2007), sauciologie (30/ 09/2007), rentrée sauciale (25/09/2007), statistoc (16/08/2007), le zite (09/07/ 2007), la batata (25/ 07/ 2007), sidi good year (28/ 07/ 2007), sidi quelque chose (28/07/2007), paralebole (09/08/2007), savoirerie (08/ 08/ 2007), une kherja (02/09/2007), une khezra (16/09/2007), une chouffa (16/ 09/2007), meussieur (28/ 07/ 2007), du fond du chœur (24/ 09/2007), aisselleblaireau (29/09/2007) ...

\section{Conclusion :}

Dans notre corpus, nous avons compté 7 émetteurs pour 140 néologismes. Ce sont tous des journalistes ayant pour langue de rédaction le français. Les cas d'inégalité dans le nombre de ces créations qu'ont révélés les résultats de la répartition selon les émetteurs, nous ont poussée à nous interroger sur les causes de ces divergences.

La comparaison des productions néologiques individuelles indique que la chronique Tranche de Vie est plus riche de néologismes que celle Rä̈na Raïkoum 
et met en évidence que le processus néologique n'est pas stable; il varie en fonction des circonstances et des conditions d'énonciation. Dans la chronique Tranche de Vie on assiste à une utilisation de plus d'un seul registre de langue. Cependant, cette réalité ne nous permet pas de déterminer avec exactitude les raisons de l'émergence des innovations lexicales dans les textes écrits par le journaliste EL GUELLIL qui pratiquait à la fois les registres courant et familier. $\mathrm{Au}$ terme de cette étude consacrée à l'analyse des innovations lexicales en discours, nous sommes arrivée au résultat que la néologie peut être favorisée par plusieurs facteurs liés aux conditions d'énonciation des néologismes. Ce qui serait également intéressant de mentionner, en guise de conclusion, c'est que le phénomène de la néologie journalistique, de par son importance qualitative et quantitative, implique les différentes disciplines de la linguistique.

Les références :

BENVENISTE E., (1974), Problèmes de Linguistique Générale, Tome2, Paris. BOYER H., (1997), Plurilinguisme: Contact ou Conflit de Langues, L'Harmattan.

BOYER H., (2001), Introduction à la Sociolinguistique, Paris, Dunod.

BROUCKER J., (1995), Pratique de L'Information et Ecritures Journalistiques, C.F.P.J.

DUCROT J. -M., «Les relations entre oral et écrit : Prise de conscience d'une problématique d'ordre didactique», Synergies F.L.E. http://www.francesynergies.org.

EURARD F., (1996), L'humour, "Contours Littéraires », Hachette.

MORTUREUX F., (1997), La Lexicologie Entre Langue et Discours, SEDES.

SABLAYROLLES J.-F., (2000), La Néologie en Français Contemporain: examen du concept et analyse de productions néologiques récentes, Paris, Honoré Champion.

VOIROL M., (1992), Guide de la Rédaction, C.F.P.J.

\section{Notes:}

(1) -BENVENISTE E., (1974), Problèmes de Linguistique Générale, Tome2, Paris, p.80.

(2)-Les chroniques dépouillées sont disponibles sur le site web du journal : www.quotidien-oran.com. 
(3) -La collecte a été faite individuellement et manuellement.

(4) -Néologisme créé par détournement de l'expression Union du Maghreb Arabe.

(5) -L'abréviation «J.» correspond au mot «journaliste». La chronique Tranche de Vie, pendant la période du dépouillement, a été animée par un seul journaliste qui est EL Guellil. Les six journalistes ayant animé la chronique Raïna Raïkoum sont: kamel Daoud, El-Houari Dilmi, Elhadj Abdelhamid, Kamel Benmohamed, Aïssa Hirèche et Mohamed Salah Boureni.

(6) -BOYER H., (1997), Plurilinguisme : Contact ou Conflit de Langues,

L'Harmattan. P. 162.

(7) - BOYER H., (2001), Introduction à la Sociolinguistique, Paris, Dunod, pp. 62-63.

(8) -BROUCKER J., (1995), Pratique de L'Information et Ecritures

Journalistiques, C.F.P.J. p.207.

(9) - VOIROL M., (1992), Guide de la Rédaction, C.F.P.J. p. 61.

(10) -MORTUREUX M.-F., (1997), La Lexicologie Entre Langue et Discours, SEDES, p. 110.

(11) - DUCROT J. -M., «Les relations entre oral et écrit : Prise de conscience d'une problématique d'ordre didactique », Synergies F.L.E. http://www.francesynergies.org. Consulté le 03/ 07/ 2008.

(12) -SABLAROLLES J. -F., (2000), La Néologie en Français Contemporain : examen du concept et analyse de productions néologiques récentes, Paris, Honoré Champion, p. 361.

(13) - SABLAROLLES J. -F., (2000),Ibid., p. 368.

(14) -SABLAYROLLES, J.-F. (2000), Ibid., p.382.

(15) - EURARD F., (1996), L'humour, « Contours Littéraires », Hachette, p.

73. 